\title{
Confined Optical Phonon Modes in Aligned Nanorod Arrays Detected by Resonant Inelastic Light Scattering
}

\author{
Concetta Nobile, ${ }^{\dagger}$ Vladimir A. Fonoberov, ${ }^{\ddagger}$ Stefan Kudera, ${ }^{\dagger}$ Antonio Della Torre, ${ }^{\dagger}$ \\ Antonio Ruffino, ${ }^{\dagger}$ Gerwin Chilla, $\$$ Tobias Kipp, $\$$ Detlef Heitmann, $\$$ \\ Liberato Manna, ${ }^{\dagger}$ Roberto Cingolani, ${ }^{\dagger}$ Alexander A. Balandin, ${ }^{\ddagger}$ and \\ Roman Krahne ${ }^{*} \dagger$
}

\begin{abstract}
National Nanotechnology Laboratory of CNR-INFM, Via per Arnesano, 73100 Lecce, Italy, Department of Electrical Engineering, University of California-Riverside, Riverside, California 92521, Institute of Applied Physics, University of Hamburg, 20355 Hamburg, Germany
\end{abstract}

Received December 2, 2006; Revised Manuscript Received January 10, 2007

\begin{abstract}
We investigated the optical phonon excitations of laterally aligned nanorod arrays by resonant Raman scattering. We observed a strong suppression of the surface-optical phonon modes in the closely packed aligned arrays and a small asymmetry in the longitudinal-optical phonon peak with respect to the rod alignment orientation. These observations can be explained by the spatial distribution of the potential of the different phonon modes derived from the first principles calculations.
\end{abstract}

The chemical synthesis of colloidal semiconductor nanorods allows one to control the length and diameter and thereby provides the possibility to tune their optical and electronic properties. For this reason, nanorods have emerged as a very interesting material for both fundamental studies ${ }^{1,2}$ and for optical ${ }^{3}$ and electronic applications. ${ }^{4}$ In particular, the uniaxial symmetry in shape and crystal structure of wurtzite nanorods has strong effects on their electronic level structure and optical excitations. ${ }^{5-8}$ This results, for example, in the emission of linearly polarized light from CdSe nanorods. ${ }^{9}$ Spectral hole-burning experiments on $\mathrm{CdSe} / \mathrm{ZnS}$ nanorods have revealed a difference in decoherence rate for rods and spherical nanocrystals by investigating the zero-phonon line line width. ${ }^{10}$ Raman spectroscopy has proven to be a powerful tool for the investigation of lattice vibrations, ${ }^{11,12}$ and confined optical phonon excitations in nanorods and nanowires have been studied experimentally ${ }^{13-19}$ and were described by different theoretical models. ${ }^{20-25}$ Recently, efforts toward the self-assembly of nanorods and nanowires into ordered arrays, for example, by electric field mediated alignment, have been reported. ${ }^{26-29}$ This technique can be exploited to realize ordered arrays of nanorods on the scale

* Corresponding author. E-mail: roman.krahne@unile.it. Telephone: +390832 298 209. Fax: +390832 298238.

$\dagger$ National Nanotechnology Laboratory of CNR-INFM.

Department of Electrical Engineering, University of CaliforniaRiverside.

$\S$ Institute of Applied Physics, University of Hamburg. of some microns, which is comparable with the spatial resolution of optical spectroscopy and thereby enables optical spectroscopy studies of orientation-dependent properties on large ensembles of nanorods.

In this letter, we present a detailed comparison between experiments and first principles theory on optical phonon excitations in CdSe nanorods. We report Raman experiments that probe the optical lattice vibrations in ordered arrays of CdSe nanorods, which were assembled on a substrate surface by electric-field-induced alignment. We found that the lateral packing of nanorods into dense arrays leads to the suppression of the surface optical phonon modes. In the LO phonon peak, we observe a fine structure that depends on the relative orientation of the nanorods with respect to the incident and detected light polarization. We compare the experimental data with first principles calculations on corresponding nanostructures, which reveal the symmetry of the phonon potentials of the Raman active modes and provide a qualitative explanation of the experimental spectra.

Colloidal wurtzite $\mathrm{CdSe}$ nanorods were fabricated by chemical synthesis in a hot mixture of surfactants following a published approach. ${ }^{30}$ The nanorods samples were inspected by transmission electron microscope imaging, and fluorescence and absorption spectra were recorded at room temperature in solution (see Supporting Information). For random orientation experiments, the nanorods were drop-casted from solution on a Si substrate and dried by nitrogen flow. 


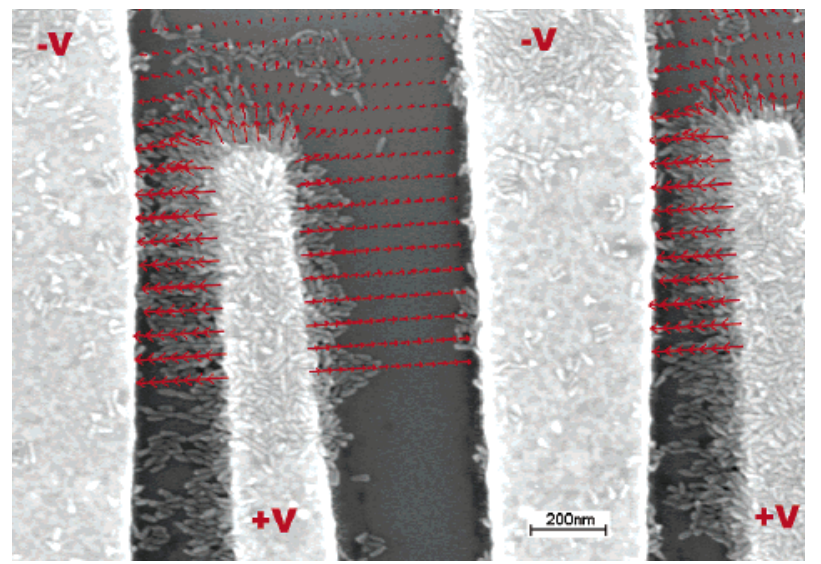

Figure 1. Scanning electron microscope image of laterally aligned nanorods in between interdigitated electrodes defined by electronbeam lithography. The red arrows show the calculated electric field for bias $\pm \mathrm{V}$ applied to neighboring electrodes (as was the case during alignment). In the lower part of the image, the electric field lines are not shown in order to enhance the visibility of the nanorods. We find that the rods accumulate in the regions of strongest electric field and observe excellent alignment with the electric field.

To laterally align nanorods, we fabricated interdigitated electrode structures with electrode distances of the order of some microns by optical lithography and metal evaporation $\left(\mathrm{Cr} / \mathrm{Au}\right.$ ) onto a $\mathrm{Si} / \mathrm{SiO}_{2}$ substrate (see schematics in Figure 2). A quantity of typically 50 microliters of nanorod solution (nanorods were dispersed in either chloroform or toluene) was drop-casted onto the electrode device, and an ac electric field of $10^{5} \mathrm{~V} / \mathrm{cm}$ at a frequency of $10 \mathrm{MHz}$ was applied during solvent evaporation. The use of alternating electric fields for the alignment avoids charging effects and accumulation of the nanorods on the electrodes. After the alignment process, the electrode devices were inspected by scanning electron microscopy (SEM) imaging (see Figure 1) and the regions of best alignment were identified for the Raman measurements. We worked with relatively large rods with $10 \mathrm{~nm}$ diameter and $50 \mathrm{~nm}$ length in order to increase their visibility in the SEM imaging. To reduce contamination of the rods due exposure to the electron beam during imaging, we worked with short exposure times and at comparatively low accelerating voltages ( $10 \mathrm{kV}$ or lower). We did not observe any influence of the SEM imaging on the line-shape of the phonons.

The samples were mounted into an optical cryostat, cooled to $T=15 \mathrm{~K}$, and the Raman experiments were performed using a diode-pumped solid-state laser at $532 \mathrm{~nm}$ wavelength that was coupled into an optical microscope setup with an $80 \times$ objective lens, which focused the light on a spot with approximately $1 \mu \mathrm{m}$ diameter. The signal was detected by the same optical path and analyzed by a triple Raman spectrometer (Dilor XY) and a charge-coupled device camera.

Figure 3a shows Raman spectra of randomly oriented (blue-dotted) and closely packed laterally aligned (red-solid) nanorods with 10/50 $\mathrm{nm}$ diameter/length that were taken on regions outside and within the interdigitated electrodes, respectively, on the same sample. In the spectrum of the

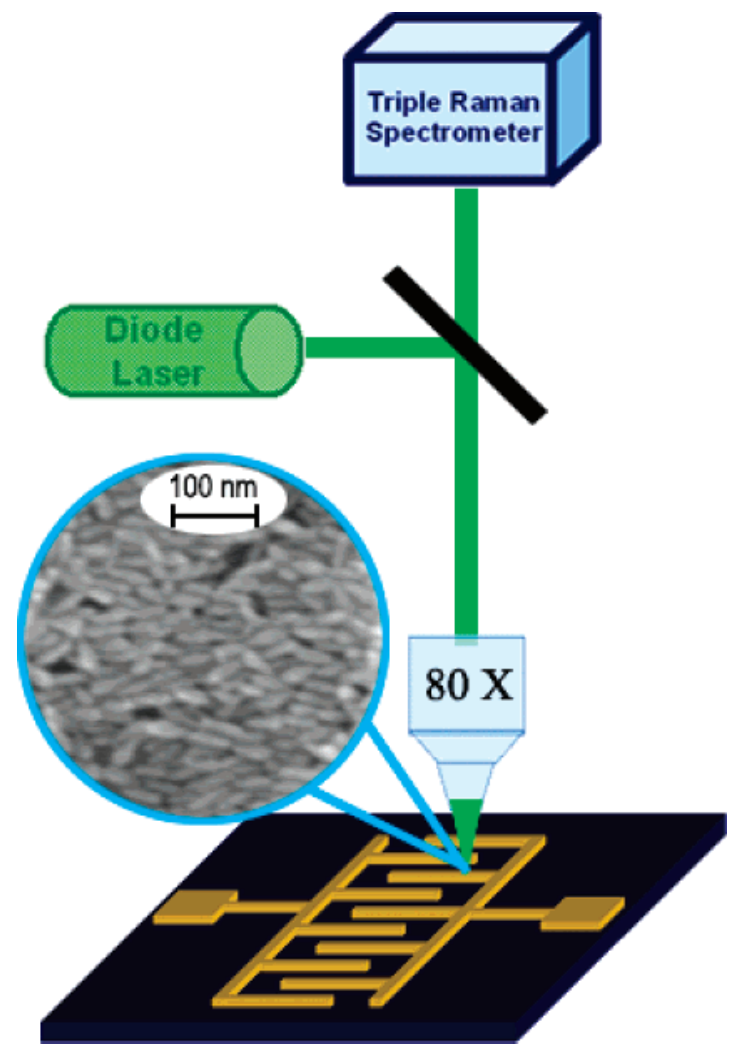

Figure 2. Schematic illustration of the experimental setup. A diodepumped solid-state laser (wavelength $532 \mathrm{~nm}$ ) was focused by an optical microscope using an $80 \times$ objective on a region of aligned nanorods in between the interdigitated electrodes. The zoom shows a SEM image of an array of aligned nanorods.

randomly oriented rods, we observe the LO phonon at 214 $\mathrm{cm}^{-1}$, and at the low-energy side, a broad peak centered around $185 \mathrm{~cm}^{-1}$ originating from the SO phonon modes. The spectrum of the laterally aligned nanorods (with $c$-axis perpendicular to the incident light polarization) shows a very small blue-shift of the LO phonon peak and a significant decrease in the SO phonon intensity. In the following, we will compare the experimental Raman spectra with first principles calculations on nanorods with corresponding aspect ratio.

The phonon modes in CdSe nanorods were calculated according to a theory developed by Fonoberov and Balandin in refs 23, 24 for spheroidal nanocrystals with wurtzite crystal lattice. It is convenient to use cylindrical coordinates $(\rho, \phi, z)$ for a spheroidal nanorod with semi-axes $a$ and $c$ and the dispersions of the anisotropic dielectric function $\epsilon_{\perp}(\omega)$ and $\epsilon_{z}(\omega)$ in the following denotations:

$$
\begin{gathered}
g_{1}=\frac{\epsilon_{z}(\omega)}{\epsilon_{\perp}(\omega)} ; \quad g_{2}=1 ; \quad f_{n}=c^{2}-g_{n} a^{2} \\
p_{n}=f_{n}+z^{2}+g_{n} \rho^{2} ; t_{n}=\sqrt{p_{n}^{2}-4 f_{n} z^{2}} \\
\xi_{n}=|z| \sqrt{\frac{2}{p_{n}-t_{n}}} ; \eta_{n}=\operatorname{sign}(z) \sqrt{\frac{p_{n}-t_{n}}{2 f_{n}}}
\end{gathered}
$$

We describe the phonon potential inside (in) and outside (out) 


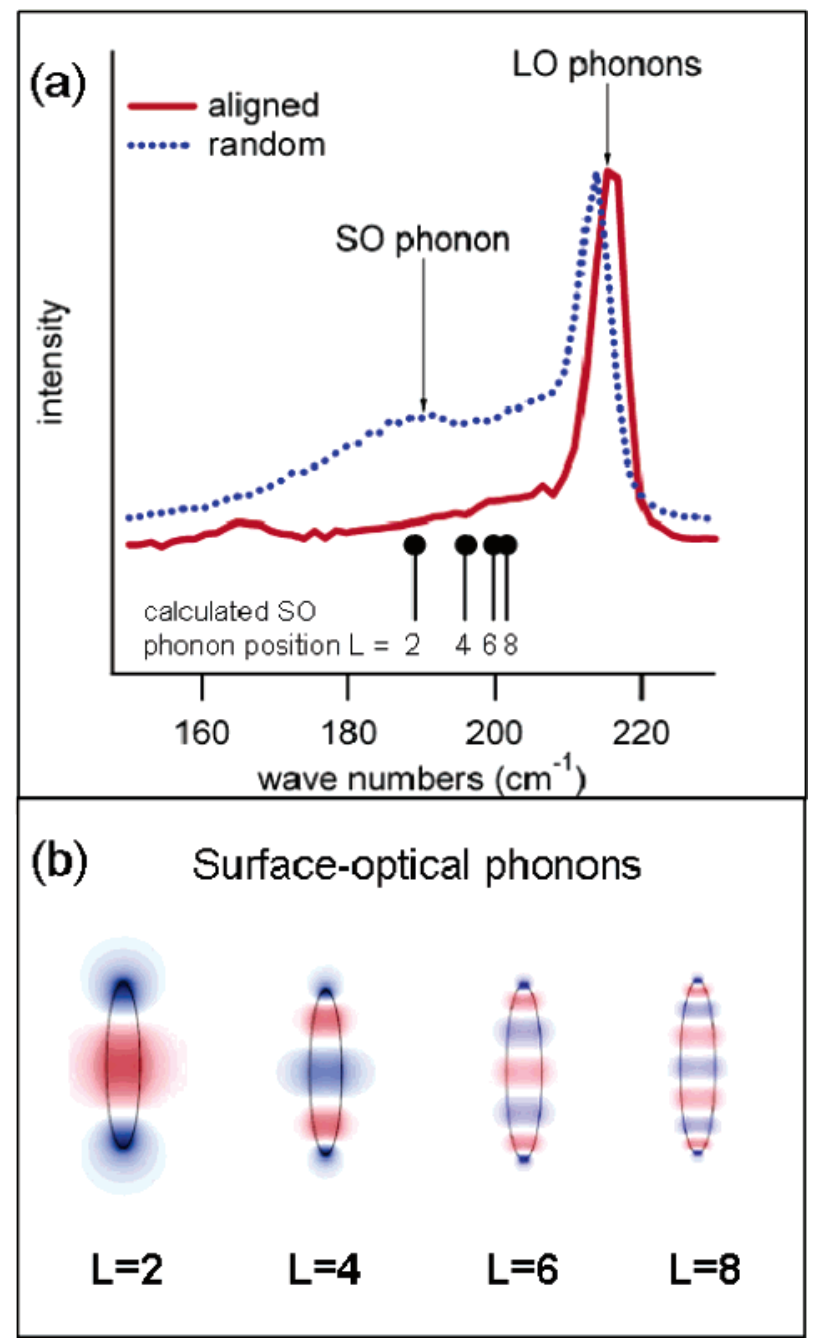

Figure 3. (a) Resonant Raman spectra of randomly oriented (bluedotted line) and laterally aligned (solid red line) nanorods at $T=$ $15 \mathrm{~K}$ recorded in parallel polarization with respect to incident and detected light. The laser energy and power density were $2.33 \mathrm{eV}$ and $2.4 \mathrm{~mW} / \mu \mathrm{m}^{2}$, respectively. The Raman signal was normalized with respect to the LO phonon peak. The black bars indicate the calculated energies for the first four Raman active SO phonon modes. (b) Cross sections of the calculated phonon potential for the SO modes with $M=0$ and different angular momentum. Only SO phonon modes with even quantum number $L$ are Raman active. Note that the $L=2$ mode has significant phonon potential outside the nanorod surface.

of the nanorod as

$$
\begin{aligned}
& V_{L, M, \omega}^{\text {(in) }}(\rho, \phi, z)=\frac{P_{L}^{M}\left(\xi_{1}\right)}{P_{L}^{M}\left(c / \sqrt{f_{1}}\right)} P_{L}^{M}\left(\eta_{1}\right) e^{i M \phi} \\
& V_{L, M, \omega}^{\text {(out })}(\rho, \phi, z)=\frac{Q_{L}^{M}\left(\xi_{2}\right)}{Q_{L}^{M}\left(c / \sqrt{f_{2}}\right)} P_{L}^{M}\left(\eta_{2}\right) e^{i M \phi}
\end{aligned}
$$

Here $P_{L}^{M}$ and $Q_{L}^{M}$ are associated Legendre functions of the first and second kind, respectively. The integers $L(L \geq 1)$ and $M(|M| \leq L)$ are quantum numbers of the phonon mode, and the eigenfrequencies $\omega$ of the phonon modes are defined from the secular eq 25 of ref 24 . In the calculation, the aspect ratio cla of $\mathrm{CdSe}$ nanorods was taken to be 5 , and the material parameters of wurtzite $\mathrm{CdSe}$ were taken from ref 31 . The theory predicts that only phonon modes with even $L$ and with $M=0$ can be active in a resonant Raman spectrum.

Figure $3 \mathrm{~b}$ shows the calculated potential of the $\mathrm{SO}$ phonon modes with $M=0$ and even angular quantum numbers $L$ according to the formalism reported above. The energy of these modes is depicted in Figure 3a by the black bars with full heads (energy is increasing with higher angular momentum number). We find excellent agreement of the calculated Raman active energies with the data obtained from the randomly oriented rods. In Figure 3b, we see that the phonon potentials of the SO modes with low $L$ extend significantly outside of the rod surface. The surface or interface phonon modes are stimulated by the change in dielectric medium at the interface. In a close-packed 2D array of nanorods, the space adjacent to the rods is occupied by other nanorods, and therefore, the dielectric medium surrounding the individual rods is severely modified, leading to the observed suppression of the SO phonon modes. The fact that SO phonon modes with higher angular momentum extend less outside the rod region accounts for the low-energy shoulder of the LO phonon peak around $200 \mathrm{~cm}^{-1}$.

Figure 4 shows spectra of aligned arrays of nanorods in which the $c$-axis of the rods was oriented parallel or perpendicular to the incident laser polarization, as illustrated by the insets. Incident laser polarization and detection were in parallel configuration. We find that the signal of the rods parallel to the laser polarization can be accurately fitted with a single Lorentz peak centered at $216 \mathrm{~cm}^{-1}$. However, in the perpendicular configuration, we observe an asymmetric peak that has to be fitted with two Lorentz peaks centered at 213.5 and $216.2 \mathrm{~cm}^{-1}$. Theory can provide a qualitative explanation for this effect: the phonon potential for the LO modes displayed in Figure $4 \mathrm{~b}$ shows that, for $L=2$ (214.9 $\left.\mathrm{cm}^{-1}\right)$ and $L=4\left(214.9 \mathrm{~cm}^{-1}\right.$ [the second mode in the $L=$ 4 pair]), the symmetry is high in both directions. Thus, such modes with high frequency are observed in both parallel and perpendicular configurations. On the other hand, lowerfrequency modes like $L=4\left(214.6 \mathrm{~cm}^{-1}\right)$ and $L=6(214.1$ $\mathrm{cm}^{-1}$ ) [the first modes of $L=4$ and $L=6$ sets in Figure 4b] have high symmetry only in the direction perpendicular to the nanorod axis. Therefore, these low-frequency LO modes have significant Raman intensity only when excited in the perpendicular configuration and are not observed in the top spectrum in Figure 4a. Calculations of LO modes with higher $L$ show that the splitting in energy between the low- and high-energy modes increases with increasing $L$; for example, the left bar in Figure 4a indicates the energy of the low-frequency mode for $L=8$ at $213.2 \mathrm{~cm}^{-1}$, and the bar on the right shows the energy of the $L=2$ (214.9 $\mathrm{cm}^{-1}$ ) mode, which is at the same energy as the high-energy mode for $L=8$. The quantitative difference between theory and experiment can be attributed to the large nanorod size that was used in the experiment in order to enhance the visibility in SEM verification of the achieved alignment. In larger nanorods, bulk effects could still contribute to the 


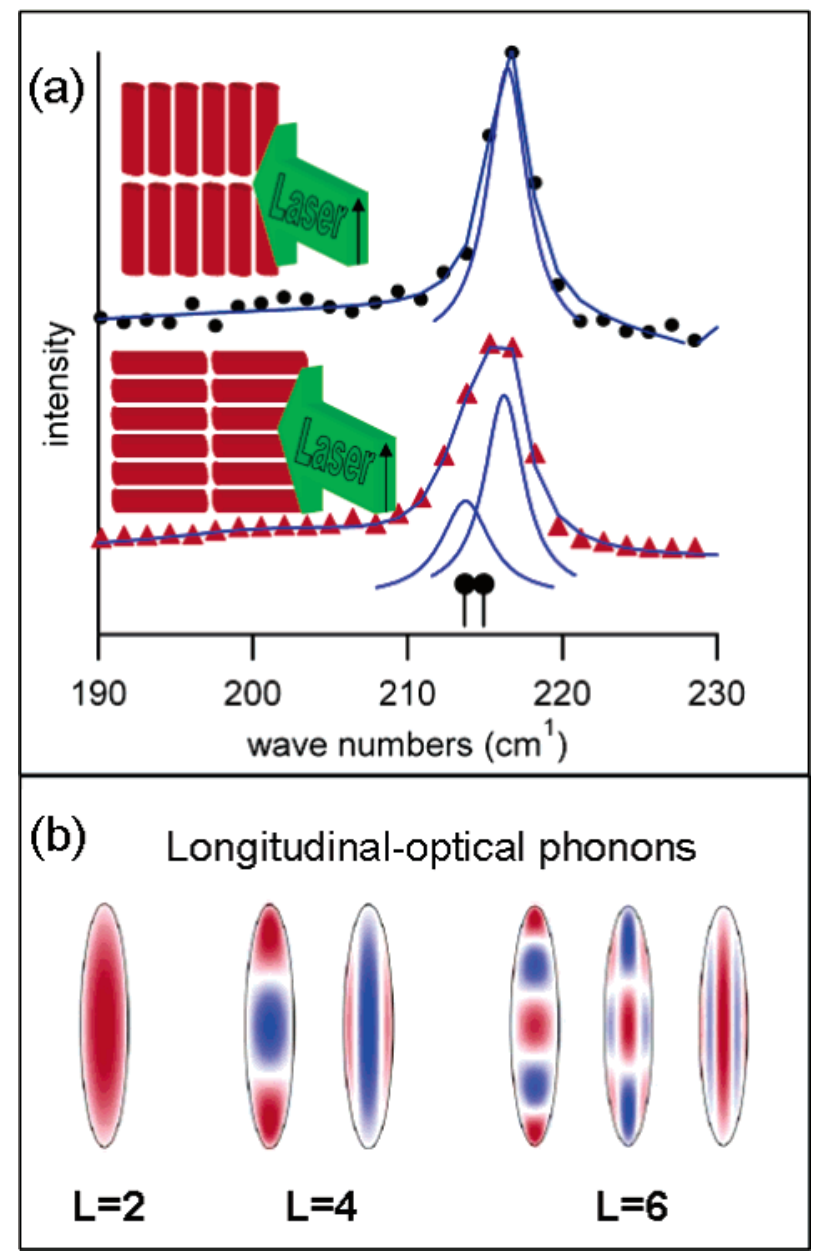

Figure 4. (a) Resonant Raman spectra of laterally aligned nanorods oriented parallel (black dots) and perpendicular (red triangles) to the incident laser polarization (as illustrated by the schematic insets where the black arrow indicates the laser polarization). The blue lines show Lorentz fits to the data and their resulting spectra. The black bars indicate the maximum splitting for $L=8$ between the low- and the high-energy mode. (b) Cross sections of the calculated phonon potential for the LO Raman-active modes with $M=0$ and different angular momentum $L$. There are $n$ Raman active LO modes for each $L=2 n$.

signal (in CdSe bulk the energy difference between the LO phonon along and perpendicular to the wurtzite $c$-axis was found to be as large as $\left.8 \mathrm{~cm}^{-1}\right) .{ }^{31}$ Therefore, bulk effects can be accounted for the large experimentally observed splitting between the low- and high-frequency modes.

In conclusion, we compared the experimental optical phonon excitations in aligned nanorod arrays with the results of first principles calculations. The observed $\mathrm{SO}$ and $\mathrm{LO}$ phonon energies are in good agreement with the theoretical predictions. In particular, the calculations yield high- and low-energy LO phonon modes that provide a qualitative explanation of the observed asymmetry in the experimental spectra with respect to the long axis orientation of the nanorods.

Acknowledgment. We gratefully acknowledge the support by the SA-NANO European project (contract no. STRP013698), by the MIUR-FIRB and MIUR 297 (contract no. 13587) projects, and by the German Science Foundation through SFB 508. The work at UCR has been supported, in part, by the UCR-UCLA-UCSB Center for Nanoscience Innovations for Defense (CNID) and NSF award to A.A.B.

Supporting Information Available: Fluorescence and absorption spectra of the nanorods. Experimental data in parallel and crossed polarization with respect to incident and detected light. This material is available free of charge via the Internet at http://pubs.acs.org.

\section{References}

(1) Li, L. S.; Hu, J. T.; Yang, W. D.; Alivisatos, A. P. Nano Lett. 2001, $1,349-351$.

(2) Li, L. S.; Alivisatos, A. P. Phys. Rev. Lett. 2003, 90, 097402.

(3) Becker, K.; Lupton, J. M.; Muller, J.; Rogach, A. L.; Talapin, D. V.; Weller, H.; Feldmann, J. Nat. Mater. 2006, 5, 777-781.

(4) Gudiksen, M. S.; Maher, K. N.; Ouyang, L.; Park, H. Nano Lett. 2005, 5, 2257-2261.

(5) Katz, D.; Wizansky, T.; Millo, O.; Rothenberg, E.; Mokari, T.; Banin, U. Phys. Rev. Lett. 2002, 89, 086801.

(6) Millo, O.; Katz, D.; Steiner, D.; Rothenberg, E.; Mokari, T.; Kazes, M.; Banin, U. Nanotechnology 2004, 15, R1-R6.

(7) Millo, O.; Steiner, D.; Katz, D.; Aharoni, A.; Kan, S.; Mokari, T.; Banin, U. Physica E 2005, 26, 1-8.

(8) Rothenberg, E.; Mokari, T.; Kazes, M.; Banin, U.; Katz, D.; Steiner, D.; Millo, O. Isr. J. Chem. 2004, 44, 391-400.

(9) Hu, J. T.; Li, L. S.; Yang, W. D.; Manna, L.; Wang, L. W.; Alivisatos, A. P. Science 2001, 292, 2060-2063.

(10) Tavenner-Kruger, S.; Park, Y. S.; Lonergan, M.; Woggon, U.; Wang, H. L. Nano Lett. 2006, 6, 2154-2157.

(11) Roca, E.; Tralleroginer, C.; Cardona, M. Phys. Rev. B 1994, 49, 13704-13711.

(12) Chamberlain, M. P.; Tralleroginer, C.; Cardona, M. Phys. Rev. B 1995, 51, 1680-1693.

(13) Teredesai, P. V.; Deepak, F. L.; Govindaraj, A.; Sood, A. K.; Rao, C. N. R. J. Nanosci. Nanotechnol. 2002, 2, 495-498.

(14) Gupta, R.; Xiong, Q.; Mahan, G. D.; Eklund, P. C. Nano Lett. 2003, $3,1745-1750$

(15) Piscanec, S.; Cantoro, M.; Ferrari, A. C.; Zapien, J. A.; Lifshitz, Y.; Lee, S. T.; Hofmann, S.; Robertson, J. Phys. Rev. B 2003, 68, 241312.

(16) Xiong, Q. H.; Chen, G.; Acord, J. D.; Liu, X.; Zengel, J. J.; Gutierrez, H. R.; Redwing, J. M.; Voon, L. C. L. Y.; Lassen, B.; Eklund, P. C. Nano Lett. 2004, 4, 1663-1668.

(17) Krahne, R.; Chilla, G.; Schüller, C.; Carbone, L.; Kudera, S.; Mannarini, G.; Manna, L.; Heitmann, D.; Cingolani, R. Nano Lett. 2006, 6, 478-482.

(18) Krahne, R.; Chilla, G.; Schüller, C.; Carbone, L.; Kudera, S.; Tarì, D.; De Giorgi, M.; Heitmann, D.; Cingolani, R.; Manna, L. J. Nanoelectron. Optoelectron. 2006, 1, 104-107.

(19) Nobile, C.; Kudera, S.; Fiore, A.; Carbone, L.; Chilla, G.; Kipp, T.; Heitmann, D.; Cingolani, R.; Manna, L.; Krahne, R. Phys. Status Solidi A 2007, http://dx.doi.org/10.1002/pssa.200673223.

(20) Mahan, G. D.; Gupta, R.; Xiong, Q.; Adu, C. K.; Eklund, P. C. Phys. Rev. B 2003, 68, 73402 .

(21) Thonhauser, T.; Mahan, G. D. Phys. Rev. B 2004, 69, 075213

(22) Thonhauser, T.; Mahan, G. D. Phys. Rev. B 2005, 71, 081307.

(23) Fonoberov, V. A.; Balandin, A. A. Phys. Rev. B 2004, 70, 233205.

(24) Fonoberov, V. A.; Balandin, A. A. J. Phys.: Condens. Matter 2005, 17, 1085-1097.

(25) Fonoberov, V. A.; Balandin, A. A. J. Nanoelectron. Optoelectron 2006, 1, 19-38.

(26) Harnack, O.; Pacholski, C.; Weller, H.; Yasuda, A.; Wessels, J. M. Nano Lett. 2003, 3, 1097-1101.

(27) Ghezelbash, A.; Koo, B.; Korgel, B. A. Nano Lett. 2006, 6, 18321836.

(28) Hu, Z. H.; Fischbein, M. D.; Querner, C.; Drndic, M. Nano Lett. 2006, 6, 2585-2591.

(29) Ryan, K. M.; Mastroianni, A.; Stancil, K. A.; Liu, H. T.; Alivisatos, A. P. Nano Lett. 2006, 6, 1479-1482.

(30) Manna, L.; Scher, E. C.; Alivisatos, A. P. J. Cluster Sci. 2002, 13, $521-532$

(31) Landolt-Boernstein; Springer-Verlag GmbH: Berlin, 1999; Vol. 41. NL062818+ 